\title{
Arzneimittelpolitik der FMH
}

\section{Tagung vom 11. November 2004, Kongresszentrum Egghölzli Bern, 13.00-16.45 Uhr}

FMH

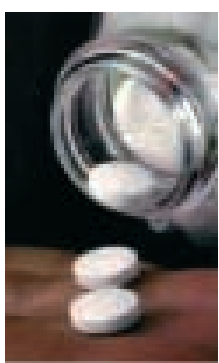

M. Giger:

P. Gyger:

K. Fattinger:

NR T. Bortoluzzi:

S. Bradke:

F. Huber:

H. H. Brunner:

Pause

Podiumsdiskussion: Wie wollen Sie Distribution und Abgabe von Arzneimitteln sicherer und effizienter gestalten? (Referentin und Referenten, Moderation U. Bucher)

M. Giger:

Begrüssung und Thesen der FMH zur Arzneimittelpolitik

Sicherheit, Patientenzufriedenheit und Wirtschaftlichkeit der Arzneimitteldistribution und Arzneimittelabgabe in der Schweiz: Beurteilung aus Sicht der Krankenversicherer

Arzneimittelsicherheit in St. Gallen und Zürich: Bericht und Empfehlungen aus Sicht der Klinischen Pharmakologen

Sicherheit, Patientenzufriedenheit und Wirtschaftlichkeit der Arzneimitteldistribution und Arzneimittelabgabe in der Schweiz: Beurteilung aus Sicht der Konsumenten

Wodurch unterscheidet sich die Patientenapotheke von der Offizinapotheke?

Die Arzneimittelabgabe im ambulanten Ärztenetzwerk, unter besonderer Berücksichtigung des Capitationmodells

Kosteneffiziente Distribution und Abgabe von pharmazeutischen Präparaten der Spezialitätenliste aus Sicht des Aufsichtsorgans

Anmeldungen bitte an Gabriela Kaufmann, Vorstandssekretariat FMH, E-Mail: gkaufman@hin.ch, Fax 03135911 12. Danke!

\section{Politique de la FMH en matière de médicaments}

\section{Colloque du 11 novembre 2004, Centre de congrès Egghölzli, Berne, 13 h 00-16 h 45}

M. Giger: Souhaits de bienvenue / Thèses de la FMH pour une politique en matière de médicaments

P. Gyger: Sécurité, satisfaction des patients et économicité de la distribution et de la dispensation des médicaments en Suisse: point de vue des assureurs-maladie

K. Fattinger: Sécurité en matière de médicaments à St-Gall et à Zurich: rapport et recommandations des pharmacologues cliniciens

T. Bortoluzzi, CN: Sécurité, satisfaction des patients et économicité de la distribution et de la dispensation des médicaments en Suisse: point de vue des consommateurs

S. Bradke: En quoi la pharmacie de cabinet médical se distingue-t-elle de la pharmacie d'officine?

F. Huber: Prescription de médicaments dans le réseau ambulatoire des médecins en cabinet privé, avec prise en compte particulière du modèle de la capitation

H. H. Brunner: Distribution et dispensation des préparations pharmaceutiques de la liste des spécialités en termes d'efficacité économique: point de vue de l'organe de surveillance

Pause

Débat: Comment organiser la distribution et la dispensation de médicaments de manière plus sûre et plus efficace? (animateur: U. Bucher)

M. Giger:

Take-Home-Messages

Prière de s'inscrire auprès de Gabriela Kaufmann, FMH, secrétariat de direction, e-mail: gkaufman@hin.ch, fax 03135911 12. Merci! 